\title{
Euclid's Fifth Postulate and Convergence of Non-Parallel Straight Lines
}

\author{
Kabenge Hamiss ${ }^{1,2}$ \\ ${ }^{1}$ Department of Mathematics and Statistics, Islamic University in Uganda, Mbale, Uganda \\ ${ }^{2}$ Department of Mathematics and Computer Science, University of Gezira, Wad-Medani, Sudan \\ Email: kabenhami@gmail.com
}

How to cite this paper: Hamiss, K. (2019) Euclid's Fifth Postulate and Convergence of Non-Parallel Straight Lines. Advances in Pure Mathematics, 9, 1059-1070. https://doi.org/10.4236/apm.2019.912052

Received: July 29, 2019

Accepted: December 21, 2019

Published: December 24, 2019

Copyright (c) 2019 by author(s) and Scientific Research Publishing Inc. This work is licensed under the Creative Commons Attribution International License (CC BY 4.0).

http://creativecommons.org/licenses/by/4.0/

\begin{abstract}
This paper proves Euclid's fifth postulate and convergence of straight lines using the formula for the area of trapezoids and assuming straight lines, it derives a general formula for the area of trapezoids involving ratios and we assume that the straight lines determine the nature and area for all the rectilinear figures. Furthermore, this proof is essential in Geometric optics basically in proving and classifying beams of light (wave) that is to mathematically prove the presence of parallel, convergent and divergent beams of light assuming the ray of light is a straight line.
\end{abstract}

\section{Keywords}

Interior Angle, Intersect, Trapezoid, Triangle, Convergence

\section{Introduction}

Euclid's first four postulates have always been readily accepted by mathematicians. The fifth postulate-the "parallel postulate"-however, became highly controversial. The fifth postulate is often called the Parallel Postulate even though it does not specifically talk about parallel lines; it actually does deal with ideas of parallelism. The consideration of alternatives to Euclid's parallel postulate resulted in the development of non-Euclidean geometries.

For more than 2000 years, mathematicians tried proving the $5^{\text {th }}$ postulate using only the first four postulates, but were unsuccessful and ended up with new geometry. Euclid's fifth postulate contributed to the development of non-Euclidean Geometry because in trying to prove it all of these mathematicians independently discovered a non-Euclidean geometry and by doing so had come to the same conclusion that the Parallel Postulate cannot be proven from the other four postulates of Euclid's Geometry. These mathematicians eventually 
discovered Hyperbolic Geometry! The first 28 theorems in Euclid's book, the Elements, do not use the parallel postulate for their proof. Some people believe the Euclid originally started off with only 4 postulates and later only added the fifth one, when he realized it would be necessary [1] [2].

Many mathematicians (amongst the Greeks, the Arabs, Europeans) believed that the 5th postulate actually was not necessary, that it could have been proven as a theorem, but up to today its proof has not been figured out [1].

Ibun Qurra formulated two propositions which are considered to be equivalent to Euclid's fifth postulate and they are "If a straight line falls on two straight lines and the two alternate angles are equal to one another then those two lines do not converge or diverge on either side." And the second proposition states that "if a straight line falls on two straight lines which do not converge or diverge on either side, then the two alternate angles are equal to one another" [3].

Euclid's Elements is by far the most famous mathematical work of classical antiquity, and also has the distinction of being the world's oldest continuously used mathematical textbook. Little is known about the author, beyond the fact that he lived in Alexandria around 300 BCE [4].

The following definitions and theorems will be of great importance in our analysis and findings, see [4] for more details.

Definition 1-1: A right angle $\mathrm{R}$ is an angle equal to its neighbouring angle on the same straight line.

Definition 1-2: Two lines are parallel if they do not intersect at any point.

Theorem 1-1: Any exterior angle is greater than any interior angle not neighbouring it.

Theorem1-2: Any two perpendiculars to a straight line are parallel.

Theorem 1-3: The sum of the angles of a triangle is $2 R$ ( $R$-right angle).

Theorem 1-4: The sum of any two neighbouring angles on the same line is $2 R$ and any two angles which are not on the same line are congruent (equal).

Theorem 1-5: The sum of any two angles of a triangle is less than $2 R$.

Therefore in these proceedings first we shall state a theorem that may be is closely related to Euclid's fifth postulate and what is considered to be its converse and then use it in the proof. In addition to that we shall assume that the nature and area of any rectilinear figure depend on the nature of the lines (dimensions).

The paper proves Euclid's fifth postulate and to show that nonparallel straight lines do not converge in the same direction they diverge.

This work is an extension of some proofs in [4] but major distinction between the results in [2], [4] and in this paper is, the geometrical constructions employed in the Elements [4] are restricted to those which can be achieved using a straight-rule and a compass, whereas in this paper the proof is based on measurement and comparison of magnitudes which is strictly forbidden in [4].

In this paper, also we derive a general formula for the area of trapezoids which may be useful in some cases and finally the proof for the convergence of nonparallel straight lines may be employed to prove the existence of divergent, parallel 
and convergent beams of light in physics.

\section{The Convergence of Nonparallel Lines and Euclid's Fifth Postulate}

We assume that the area of the rectilinear figure formed is the sum of the areas of the trapezoids when the figure is split into several portions (trapezoids). This assumption is backed up by the postulates (17) and (19) in [5].

In this paper, we take the simplest and basic type of region, the trapezoidal region and we use it as the building block to define the idea of the rectilinear figure formed in the problem we are yet to solve [6]. So the basic procedure of solving this is to split the figure into trapezoidal regions and this will provide us with the best basis for our theory. In addition to that, the trapezoidal regions must not overlap that is they must not have any trapezoidal region in common but they may have only a common point or a common segment. If we permit the regions to overlap we cannot say that the area of the figure is the sum of the areas of its component trapezoidal regions. In this case our treatment of the area is similar to that for distance and measure angle.

However, to be quite specific we shall not consider the sum directly but rather we shall check on the convergence of the sequence of areas of the trapezoids and if the sequence converges to zero then, it implies the convergence of nonparallel lines.

\section{Results}

Euclid's fifth postulate states that "if a straight line intersects two straight lines such that the sum of the interior angles of intersection is less than two right angles then the two lines must intersect on the side of the interior angles". In this section we first reformulate Euclid's fifth postulate and prove it in a more general way.

\section{Proposition 3-1:}

If a line intersects two lines at different points, the sum of the interior angles of intersection is less than $2 R$ if and only if a triangle can be produced on the side of the interior angles.

Proof: Let the line $c$ intersect two lines $a$ and $a^{\prime}$ at points A and B respectively such that the lines $a$ and $a$ also intersect at point $C$ to form a triangle as in Figure 1.

From Figure 1 and using theorem 1-1, it's we observe that

$$
\alpha<\beta_{1}
$$

but $\beta+\beta_{1}=2 R$ from theorem 1-4 where $\beta_{1}$ an exterior angle is supplementary angle to $\beta$ and $R$ is a right angle.

$$
\beta_{1}=2 R-\beta
$$

From expressions (1) and (2) therefore 


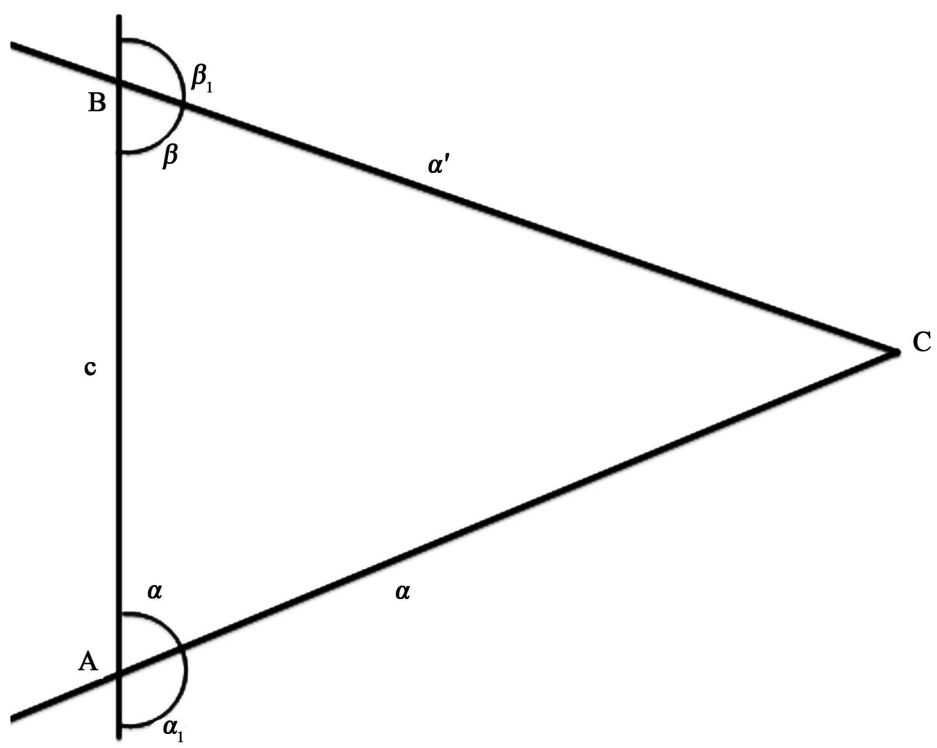

Figure 1. Triangle formed.

$$
\alpha<2 R-\beta
$$

hence $\alpha+\beta<2 R$ thus proved.

This implies that when a straight line $c$ intersects two intersecting straight lines $a$ and $a$ forming a triangle then the sum of the interior angles of intersection of lines $a$ and $a$ with $c$ is less than $2 R$. This proves the converse part of theorem 2-1.

However, our main goal in this paper is to prove the remaining part of this theorem. That is to prove that if the sum of the interior angles of intersection is less than $2 R$, then the two lines must intersect to form a triangle on that same side of the interior angles of intersection when straight lines $a$ and $a$ are extended indefinitely. Now we prove the converse part of the theorem: Let line $c$ intersect two lines $a$ and $a$ at $\mathrm{A}$ and $\mathrm{B}$ respectively such that the sum of the interior angles of intersection is less than $2 R$

$$
\alpha+\beta<2 R
$$

From Figure 2 let $\alpha_{1}$ and $\beta_{1}$ be the exterior supplementary angles to $\alpha$ and $\beta$ respectively along line $c$.

We already know that the sum of $\alpha$ and $\beta$ is less than $2 R$ from (3).

Now, we assume the opposite that the lines $a$ and $a$ do not intersect at any point.

Then lines $a$ and $a$ are parallel to one another, then we have the following possibilities:

Possibility 1:

$a$ and $a$ are perpendicular to line $c$

Now, using theorem 1-4 then

$$
\alpha+\alpha_{1}=2 R
$$

and 


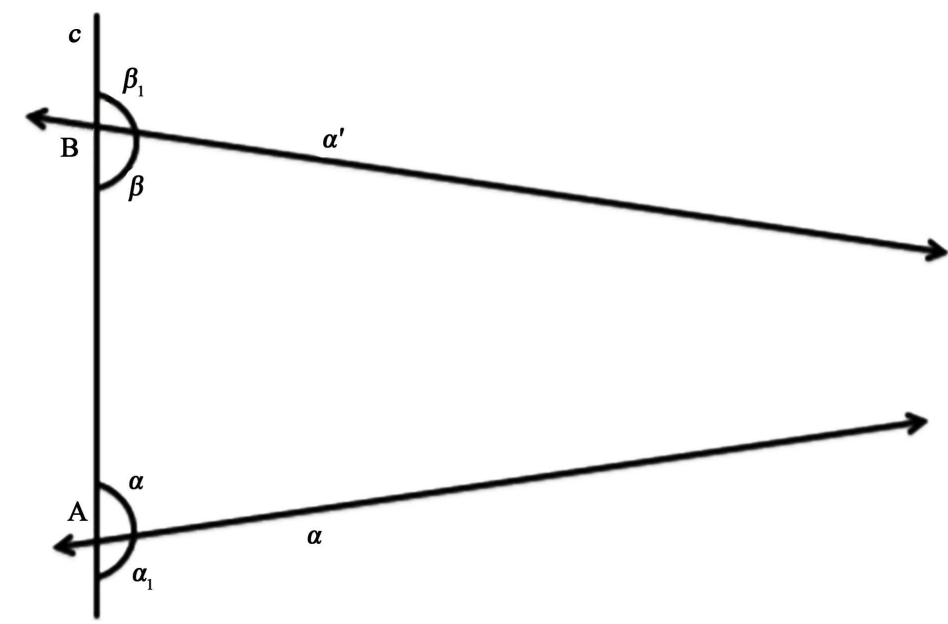

Figure 2. Straight lines $a$ and $a$ 'not intersecting anywhere.

$$
\beta+\beta_{1}=2 R
$$

Adding the two Equations (4) and (5) we obtain

$$
\begin{aligned}
& (\alpha+\beta)+\left(\alpha_{1}+\beta_{1}\right)=4 R \\
& \left(\alpha_{1}+\beta_{1}\right)=4 R-(\alpha+\beta)
\end{aligned}
$$

Since $\alpha+\beta<2 R$ then

$$
\alpha_{1}+\beta_{1}>2 R
$$

This clearly shows that from inequalities have three cases in each For

$$
\alpha+\beta<2 R
$$

Case 1

If

$$
\alpha=R,
$$

then

$$
\beta<R
$$

Case 2

If

$$
\beta=R
$$

then

$$
\alpha<R
$$

Case 3

If

$$
\alpha=\beta
$$

then

$$
\alpha=\beta<R
$$

Similarly 
For

$$
\alpha_{1}+\beta_{1}>2 R
$$

Case I

If

$$
\alpha_{1}=R
$$

then

$$
\beta_{1}>R
$$

Case II

If

$$
\beta_{1}=R,
$$

then

$$
\alpha_{1}>R
$$

Case III

$$
\alpha_{1}=\beta_{1}>R
$$

The above inequalities from the six cases clearly contradict the theorem of parallel lines Theorem 1-2 (Our assumption) that is $\alpha \neq \beta \neq \alpha_{1} \neq \beta_{1} \neq R$ which implies that the two lines $a$ and $a$ are not parallel.

Possibility 2:

If the line $c$ is not perpendicular to the parallel lines $a$ and $a$ then we get the alternate angles that is

$$
\begin{gathered}
\alpha_{1}+\alpha=2 R \\
\beta+\beta_{1}=2 R
\end{gathered}
$$

But

$$
\alpha+\beta<2 R
$$

then let there exist a real number $\epsilon>0$ such that

$$
\alpha+\beta+\epsilon=2 R
$$

Now subtracting Equation (c) from (a) and (b) respectively, we obtain

$$
\alpha_{1}-\beta=\epsilon
$$

and

$$
\beta_{1}-\alpha=\epsilon
$$

Implying $\beta_{1}>\alpha$ and $\alpha_{1}>\beta$ which contradicts the alternate angle theorem.

From the two possibilities it implies the lines $a$ and $a$ are not parallel provided the sum of two interior angles is less than $2 R$.

Furthermore, using Figure 3 below, let us assume that $a$ and $a$ intersect at point $\mathrm{D}$ to form a triangle on the left hand side of line $c$.

Then the exterior angle $\alpha$ is less than its non-neighbouring interior angle $\beta_{1}$ which contradicts the exterior angle theorem 1-1. This implies that if the 


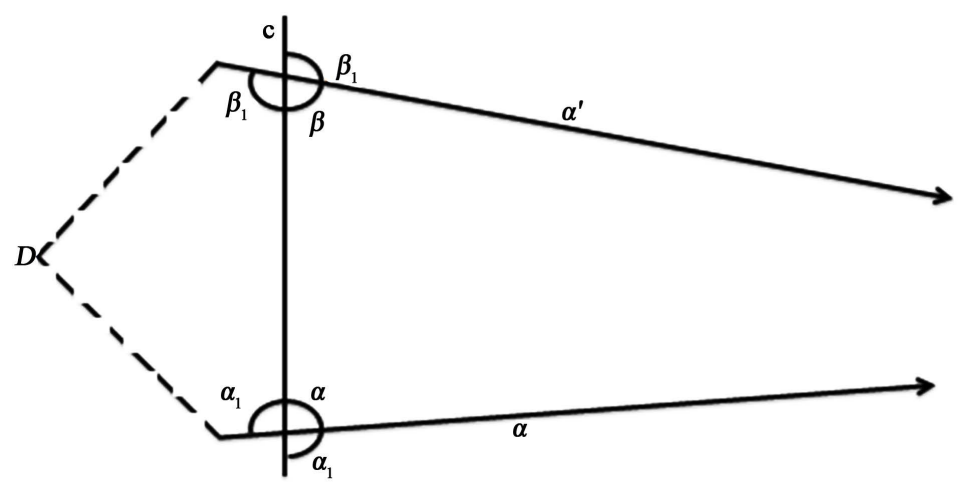

Figure 3. Straight lines $a$ and $a$ 'intersecting at D.

three lines $c$, $a$ and $a$ intersect to form a triangle, then on the right hand side of line $c$. We also know from the theorem 1-5 and since

$$
\alpha+\beta<2 R,
$$

we claim that these two angles $\alpha$ and $\beta$ are angles of a triangle on the right hand side of line $c$.

So we need to determine a point on the right hand side of line $c$ at which the two lines $a$ and $a$ intersect.

Now we construct parallel lines to line $c$ as in Figure 4 below and keeping a uniform interval between any two preceding trapezoids, we obtain a sequence of trapezoids with decreasing areas.

Because lines $a$ and $a$ are not parallel, let trapezoid 1 of area $A_{1}$ be much larger on its far left than its right hand side and its right hand side is equal to the left hand side of trapezoid 2 of area $A_{2}$ and the left hand side of trapezoid 2 is larger than its right hand side which in turn is equal to left hand side of trapezoid 3 with area $A_{3}$ and this continues up to the $n^{\text {th }}$ trapezoid and so on.

This implies that the areas of trapezoids will be $A_{1}>A_{2}>\cdots>A_{n}>\cdots$ as seen in Figure 4.

Now applying mid segment formula for the lengths of trapezoids we obtain the lengths in terms of $l_{o}$ of the first trapezoid and the length $l_{n}$ of the $n^{\text {th }}$ trapezoid.

Now to prove the

$$
\begin{aligned}
& l_{1}=\frac{1}{2}\left(l_{o}+l_{2}\right) \\
& l_{2}=\frac{1}{2}\left(l_{1}+l_{3}\right)
\end{aligned}
$$

Substituting Equation (7) in to Equation (8) we obtain

$$
l_{2}=\frac{1}{2}\left(\frac{1}{2}\left(l_{o}+l_{2}\right)+l_{3}\right)
$$

then

$$
l_{2}=\frac{1}{3} l_{o}+\frac{2}{3} l_{3}
$$




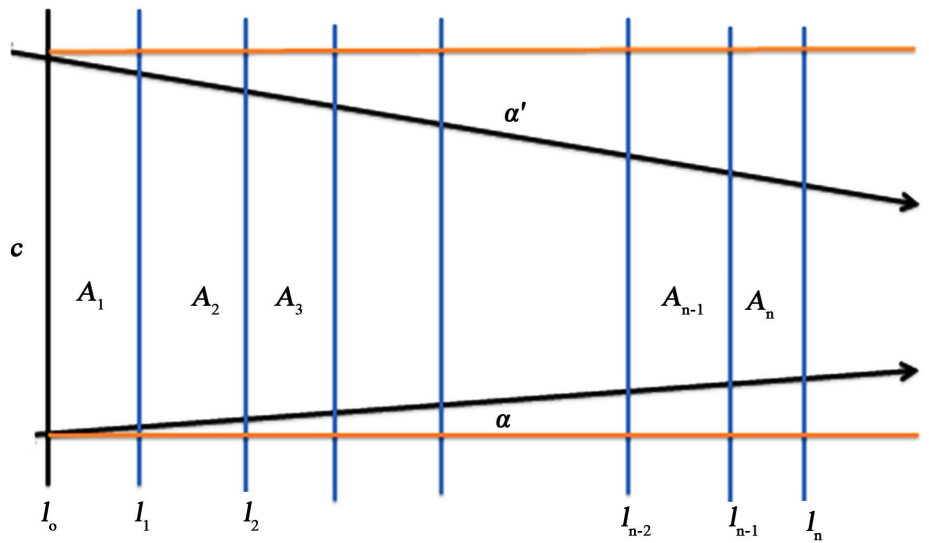

Figure 4. Parallel lines to line $\mathrm{c}$ at equal intervals.

$$
\begin{gathered}
l_{3}=\frac{1}{2}\left(l_{2}+l_{4}\right)=\frac{1}{2}\left(\frac{1}{3} l_{o}+\frac{2}{3} l_{3}+l_{4}\right) \\
l_{3}=\frac{1}{4} l_{o}+\frac{3}{4} l_{4}
\end{gathered}
$$

From the equations above we can deduce the general formula for the $n^{\text {th }}$ trapezoid of length $l_{n}$

$$
l_{n}=\frac{1}{n+1} l_{o}+\frac{n}{n+1} l_{n+1}
$$

From Figure 4 above, let the length of each interval be $d$, then we can find the areas of trapezoids.

Therefore

$$
A_{1}=\frac{1}{2}\left(l_{o}+l_{1}\right) d
$$

$A_{2}=\frac{1}{2}\left(l_{1}+l_{2}\right) d$ but $l_{1}=\frac{1}{2}\left(l_{o}+l_{2}\right)$, we now write $A_{2}$ in terms of $l_{o}$ and $l_{2}$, implying that

$$
A_{2}=\frac{1}{2 \times 2}\left(l_{o}+3 l_{2}\right) d
$$

We obtain $A_{3}$ from

$$
A_{3}=\frac{1}{2}\left(l_{2}+l_{3}\right) d
$$

But we have $l_{2}=\frac{1}{3} l_{o}+\frac{2}{3} l_{3}$, therefore substituting $l_{2}$ in Equation (14) we obtain

$$
A_{3}=\frac{1}{2 \times 3}\left(l_{o}+5 l_{3}\right) d
$$

In the same way we can obtain up to $A_{n}$ given by

$$
A_{n}=\frac{1}{2 \times n}\left(l_{o}+(2 n-1) l_{n}\right) d
$$


where $n=1,2,3, \cdots$.

Since $n=1,2,3, \cdots$ is a discrete, we shall obtain a sequence of areas of different sizes of different trapezoids. Then

$$
\left\{A_{1}, A_{2}, A_{3}, \cdots, A_{n}, \cdots\right\}=\left\{A_{n}\right\}_{n=1}^{\infty}
$$

But also from Equations (16) and (17) we obtain

$$
\left\{A_{n}\right\}_{n=1}^{\infty}=\left\{\frac{1}{2 \cdot n}\left(l_{o}+(2 n-1) l_{n}\right) d\right\}_{n=1}^{\infty}
$$

To determine the intersection of lines $a$ and $a$ we show that the sequence in Equation (18) converges and it must converge to zero, otherwise the two lines a and $a^{\prime}$ do not intersect.

Since we know that $l_{o}>l_{1}>l_{2}>\cdots>l_{n}>\cdots$ and $l_{n}$ 's are parallel to one another in a uniform interval $d$.

Now let for each $l_{n}$ there exist a real number $\lambda_{n} \in(0,1)$ such that:

$$
\lambda_{n}=\frac{l_{n}}{l_{n-1}}
$$

Equation (19) implies

$$
\lambda_{n} l_{n-1}=l_{n}
$$

From Equation (20) we obtain

$$
\begin{aligned}
& \lambda_{1} l_{o}=l_{1} \\
& \lambda_{2} l_{1}=l_{2} \\
& \lambda_{3} l_{2}=l_{3}
\end{aligned}
$$

We can obtain up to the $n^{\text {th }}$ trapezoid that is

$$
\lambda_{n} l_{n-1}=l_{n}
$$

Expressing $l_{n}$ in terms of $l_{o}$ and $\lambda_{n}$ we obtain

$$
\lambda_{1} l_{o}=l_{1} \quad \lambda_{2} l_{1}=l_{2},
$$

but $\lambda_{1} l_{o}=l_{1}$ implying that

$$
\begin{gathered}
\lambda_{2} \lambda_{1} l_{o}=l_{2} \\
\lambda_{3} \lambda_{2} \lambda_{1} l_{o}=l_{3}
\end{gathered}
$$

We obtain up to $l_{n}$ in a similar way

$$
\prod_{k=1}^{n} \lambda_{k} l_{o}=l_{n}
$$

Substituting Equation (25) in to Equation (18) we obtain

$$
\left\{A_{n}\right\}_{n=1}^{\infty}=\left\{\frac{1}{2 \cdot n}\left(l_{o}+(2 n-1) \prod_{k=1}^{n} \lambda_{k} l_{o}\right) d\right\}_{n=1}^{\infty}
$$

Therefore

$$
\left\{A_{n}\right\}_{n=1}^{\infty}=l_{o} d\left\{\frac{1}{2 \cdot n}\left(1+(2 n-1) \prod_{k=1}^{n} \lambda_{k}\right)\right\}_{n=1}^{\infty}
$$


In limits

$$
\lim _{n \rightarrow \infty} A_{n}=l_{o} d \lim _{n \rightarrow \infty} \frac{1}{2 \cdot n}\left(1+(2 n-1) \prod_{k=1}^{n} \lambda_{k}\right)=0
$$

Equation (26) implies that as $n$ grows bigger, $A_{n}$ tends to zero which clearly shows that the lines $a$ and $a^{\prime}$ intersect.

This comes from the fact that $\prod_{k=1}^{n} \lambda_{k}$ is a product of numbers between 0 and 1 so as $n$ extends to infinity, $\prod_{k=1}^{n} \lambda_{k}$ goes to zero and $\frac{1}{2 \cdot n}$ goes to zero.

Also, let's assume $\lambda_{1}=\lambda_{2}=\lambda_{3}=\cdots=\lambda_{n}=\lambda \in(0,1)$, then

$$
\prod_{k=1}^{n} \lambda_{k}=\lambda^{n}
$$

Substituting Equation (27) into Equation (25) we obtain

$$
\left\{A_{n}\right\}_{n=1}^{\infty}=l_{o} d\left\{\frac{1}{2 \cdot n}\left(1+(2 n-1) \lambda^{n}\right)\right\}_{n=1}^{\infty}
$$

In limits again we obtain

$$
\lim _{n \rightarrow \infty}\left\{A_{n}\right\}_{n=1}^{\infty}=l_{o} d \lim _{n \rightarrow \infty} \frac{1}{2 \cdot n}\left(1+(2 n-1) \lambda^{n}\right)=0
$$

Equations (26) and (28) agree in limits. This implies the lines $a$ and $a$ intersect (converge) at some point with area approximately zero.

We need to verify whether this is the only condition through which the lines a and $a^{\prime}$ intersect.

Let us consider Figure 2 above. Assume $a$ and $a$ are parallel to each other, then

$$
\lambda_{1}=\lambda_{2}=\lambda_{3}=\cdots=\lambda_{n}=\lambda=1
$$

This means $l_{o}=l_{1}=l_{2}=\cdots=l_{n}=\cdots$

Substituting Equation (30) into Equation (28) we obtain

$$
\left\{A_{n}\right\}_{n=1}^{\infty}=l_{o} d\left\{\frac{1}{2 \cdot n}\left(1+(2 n-1) \lambda^{n}\right)\right\}_{n=1}^{\infty}=l_{o} d\left\{\frac{1}{2 \cdot n}(1+(2 n-1))\right\}_{n=1}^{\infty}
$$

In limits

$$
\lim _{n \rightarrow \infty} A_{n}=l_{o} d
$$

which gives the area of a rectangle. This indeed indicates that if $\lambda_{1}=\lambda_{2}=\lambda_{3}=\cdots=\lambda_{n}=\lambda=1$, then $l_{o}=l_{1}=l_{2}=\cdots=l_{n}=\cdots$ hence giving us rectangles of the same sizes because $d$ is uniform which clearly shows that the sequence of areas is of the form $A=\left\{l_{o} d, l_{o} d, \cdots, l_{o} d, \cdots\right\}$.

On the other hand using Figure 5 below, let's assume the lines $a$ and $a$ 'intersect on the left hand side of line $c$, then

$$
1<\lambda_{1}<\lambda_{2}<\lambda_{3}<\cdots<\lambda_{n}<\cdots
$$

Implying $l_{o}<l_{1}<l_{2}<l_{3}<\cdots<l_{n}<\cdots$ then

$$
\left\{A_{n}\right\}_{n=1}^{\infty}=l_{o} d\left\{\frac{1}{2 \cdot n}\left(1+(2 n-1) \prod_{k=1}^{n} \lambda_{k}\right)\right\}_{n=1}^{\infty}
$$




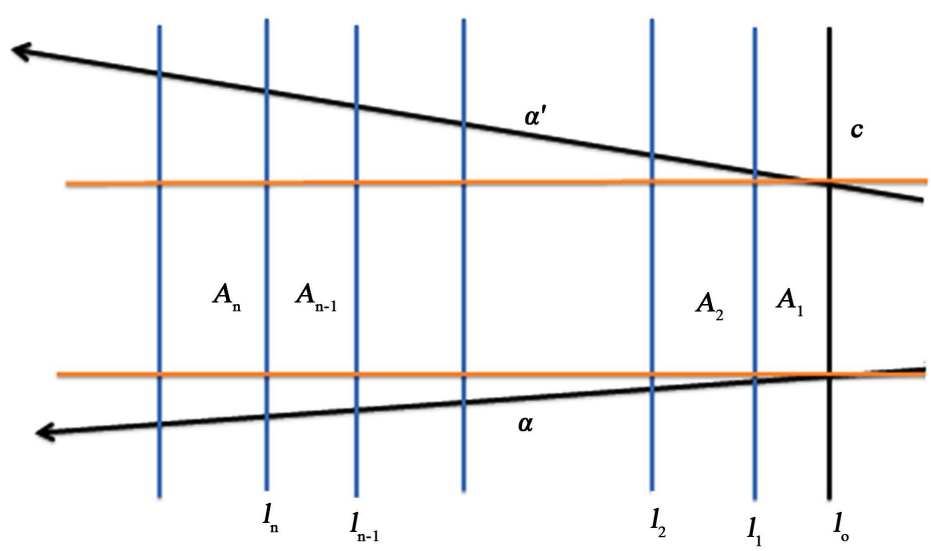

Figure 5. Straight lines $a$ and $a$ 'not intersecting on the right hand side of line $c$.

Equation (32) diverges because $\prod_{k=1}^{n} \lambda_{k}$ goes to infinity as $n$ grows bigger. Similarly as in Equation (27) if $1<\lambda_{1}=\lambda_{2}=\lambda_{3}=\cdots=\lambda_{n}=\cdots=\lambda$.

Equation (32) becomes

$$
\left\{A_{n}\right\}_{n=1}^{\infty}=l_{o} d\left\{\frac{1}{2 \cdot n}\left(1+(2 n-1) \lambda^{n}\right)\right\}_{n=1}^{\infty}
$$

In limits Equation (33) diverges as $n$ grows bigger. This is because $\lambda^{n} \rightarrow \infty$ as $n \rightarrow \infty$ due to the fact that $\lambda>1$ which indeed shows that the sequence is a strictly increasing sequence such that $A_{n}<A_{n+1}$.

As we obtained in Equations (26) and (29) now we need to determine whether when lines $a$ and $a$ intersect to form a triangle or not when they intersect with line $c$.

Let $\mathrm{E}$ be the point of intersection of lines $a$ and $a^{\prime}$ line through $\mathrm{E}$ parallel to line $c$ has $\lambda=0$, now taking $\lambda=0$ and $n=1$ Equation (28) becomes

$$
A_{1}=\frac{l_{o} d}{2 \times 1}(1+0)=\frac{l_{o} d}{2}
$$

Equation (34) gives us the area of a triangle, where $l_{o}$ is the base and $d$ is the height of the triangle.

This proves that if the sum of the interior angles of intersection of line $c$ with lines $a$ and $a^{\prime}$ is less than $2 R$, then the lines $a$ and $a^{\prime}$ intersect to form a triangle.

Hence proving that the lines $a$ and $a$ intersect at point E thus Euclid's fifth postulate.

\section{Conclusions}

In attempting to prove Euclid's fifth postulate using the first four postulates, this led to the emergence of Non-Euclidean Geometry which is useful in various fields like engineering and science. This indeed proves the strength of Euclid's parallel postulate in the real world.

This result can be applied in proving the convergent, parallel and divergent bean of light both in 2 dimensional planes and 3 dimensional spaces (here we can apply the idea of cone and spherical shaped beam of light). 


\section{Acknowledgements}

Thanks Ass Prof. Mogtaba Mohammed Yousif and Prof. Nafiu Lukman Abiodun More for many helpful discussions and comments on the manuscript and the Department of Mathematics and Statistics (IUIU) for the encouragement and support.

\section{Conflicts of Interest}

The author declares no conflicts of interest regarding the publication of this paper.

\section{References}

[1] Greenberg, M.J. (2008) Euclidean and Non-Euclidean Geometries-Development and History. Frontiers in Mathematics. W. H. Freeman, New York.

[2] Diaz, J.E. (2018) Fifth Postulate of Euclid and the Non-Euclidean Geometries. International Journal of Scientific and Engineering Research, 9, 530.

[3] Rosenfeld, B.A. (2012) A History of Non-Euclidean Geometry. Springer-Verlag New York Inc., New York.

[4] Euclid, J.L. and Fitspatrick, R. (2008) Euclid's Elements of Geometry. Fitspatrick, R., Ed. University of Texas, Austin.

[5] Allen, F.B. (1960) School Mathematics Study Group-Geometry Part II. Yale University Press, New Haven.

[6] Salilew, G.A. (2017) New Approach for Similarity of Trapezoids, Global Journal of Science Frontier Research: F Mathematics and Decision Sciences, 17, 2249-4626. 